\title{
Covid-19 and Parkinson's disease: an overview
}

\author{
S. M. Cartella ${ }^{1}$ (1) $\cdot$ C. Terranova ${ }^{1} \cdot$ V. Rizzo ${ }^{1} \cdot$ A. Quartarone $^{1} \cdot$ P. Girlanda ${ }^{1}$
}

Received: 29 December 2020 / Revised: 26 June 2021 / Accepted: 20 July 2021 / Published online: 27 July 2021

(c) Springer-Verlag GmbH Germany, part of Springer Nature 2021

\begin{abstract}
In March 2020, WHO declared Covid-19 outbreak pandemic. There has been increasing evidence that frail, old, multi-pathological patients are at greater risk of developing severe Covid-19 infection than younger, healthy ones. Covid-19's impact on Parkinson's Disease (PD) patients could be analysed through both the influence on PD patients' health and their risk of developing severe Covid-19, and the consequences of lockdown and restrictive measures on mental and cognitive health on both patients and caregivers. Moreover, there are critical issues to be considered about patients' care and management through an unprecedented time like this. One important issue to consider is physiotherapy, as most patients cannot keep exercising because of restrictive measures which has profoundly impacted on their health. Lastly, the relationship between PD and Sars-Cov2 may be even more complicated than it seems as some studies have hypothesized a possible Covid-19-induced parkinsonism. Hereby, we review the state of the art about the relationship between Covid-19 and Parkinson's Disease, focusing on each of these five points.
\end{abstract}

Keywords Parkinson's disease $\cdot$ Covid-19 $\cdot$ Movement disorders

\section{Introduction}

The last 20 years have been plagued by novel viral epidemics such as severe acute respiratory syndrome (SARS) and Middle Eastern respiratory syndrome (MERS). These conditions are characterized by a significant mortality with only few therapeutic options available. A new coronavirus infection outbroke in China in 2019, becoming pandemic in March 2020. It manifests as a respiratory infection characterized by dyspnea, fever and cough [1]. Most patients have a mild or moderate infection, even if a minority of them needs hospitalization and sometimes intubation for severe respiratory distress [2]. Covid-19 may spread beyond the respiratory system [3] causing acute kidney failure, a coagulopathy reminiscent of intravascular coagulation [4] and thrombosis [5], eventually causing multi-organ failure [6]. A substantial number of Covid-19 patients may develop neurological manifestations due to CNS (headache, fatigue, stroke, dizziness, syncope, seizures) [7] and peripheral nervous system (ageusia, anosmia, neuropathic pain and myalgias) involvement

S. M. Cartella

sandycartella92@gmail.com

1 Department of Clinical and Experimental Medicine, University of Messina, Messina, Italy
[8]. The involvement of CNS and PNS is independent of the severity of the respiratory disease [9].

On the other hand, Covid-19 may influence the clinical course of several chronic neurological conditions, especially in elderly and frail patients including Parkinsonian patients. This is a narrative review on the impact of Covid-19 on patients affected by Parkinson's Disease (PD), regarding its clinical and psychological impact, as well as the management of such patients.

\section{Clinical impact}

There has been increasing evidence of the association of Covid-19 and old, frail, multi-pathological patients [2]. PD patients are often elderly ones and they have comorbidities and advanced disease complications, among which pneumonia and respiratory failure [10, 11]. Moreover, anti-Covid-19 measures such as self-isolation and lockdown, increased depression and anxiety, have made it impossible for patients to practice physiotherapy [11]. Conversely, some factors can protect PD patients from developing severe Covid-19, starting from its molecular pathophysiology. Up to $65 \%$ of patients affected by Covid-19 present with hyposmia, which is also a common feature of Parkinson's Disease [9]. This 
may only be an intriguing coincidence [10], as Sars-Cov2 direct invasion of the brain has been hypothesized. CSF expression of Sars-Cov2 RNA has been found in one patient, but has failed to be demonstrated in others [9]. What has indeed been proved is the an activation of both innate and adaptive immune response [9], as it happens in Parkinson's Disease [12]. Alpha-synuclein (alpha-syn) is upregulated after immune stimulation [12] and it restricts the replication of RNA viruses and protects mice from virus-induced neuronal injury [12]. It has been hypothesized that alpha-syn inhibits the replication of viruses from PNS to CNS [12]. Moreover, it is involved in the activation of microglial cells $[12,13]$. Future studies are needed to better understand the interplay between alpha-syn and the immune system.

PD itself is not a risk for developing severe Covid-19. Some studies have pointed out that cardiovascular comorbidities are the actual risk [10]11,14-16, [17] [18].

Sedentary lifestyle can worsen both PD and cardiovascular diseases. Diabetes, another recognized risk factor for severe Covid-19, is also a frequent comorbidity of PD [10].

There is a subgroup of PD patients with respiratory dysfunction [11,16]. that is observed in almost $40 \%$ of PD patients without a history of lung or cardiac disease [10]. It is common in advanced PD because of camptocormia, rigidity of respiratory muscles, dysphagia, impairment of cough reflex, axial manifestations, causing poor respiratory excursion and eventually ventilatory failure [10], 11. In the early phases of the disease, there is a group of patients with respiratory failure, due to brainstem involvement. Brainstem, as recalled above, is a target of Sars-Cov2, therefore PD patients exposed to it may be particularly prone to develop respiratory failure [10].

Some studies [11, 14, 19], highlight the atypical presentation of Covid-19 in PD patients. Like all kinds of infection, it can worsen motor and non-motor symptoms, or cause the appearance of some symptoms never experienced before, such as hallucination or disabling dyskinesias [18]. In one study [11], 50\% of patients presented with atypical symptoms, such as delirium. This study, and other ones, $[14,16]$ [18] also point out there is often need to increase dopamine replacement therapy during the infection (in this study in almost half of the patients [11]). It can also occur a suboptimal absorption of oral therapy due to diarrhoea [14]. As far as device-aided therapies are concerned, Antonini et al. [11] point out the mortality rate they found in patients treated with such therapies (50\%) is not due to the therapies themselves, but to the older age and major comorbidities of the patients.

Another study based on telephonic interview of 1486 PD patients in Lombardy, Italy, identified $7.1 \%$ of them positive to Covid-19 [20]. Compared to unaffected PD patients, they were younger, accordingly with what was found in another study [14] (probably because of more restrictive measures used with older people), more likely to suffer from obstructive pulmonary disease, obese and vitamin D non-supplemented [20]. 7.6\% of caregivers were diagnosed with Covid-19, and the only differences with PD patients were the age and the higher number of weekly outings. In this study, the incidence of Covid-19 was similar between PD patients and family controls, confirming PD is not a risk factor for Covid-19, accordingly with the findings of another study [18]. The clinical manifestations of Covid-19 were in line with the study quoted above [11], except for the incidence of shortness of breath [20]. This study found a mortality rate of $5.7 \%$, that did not significantly differ from the age-matched general population's one. A multicentric study found an overall mortality in PD patients affected by Covid- 19 of $20 \%$ [16], confirming the role of advanced age and cardiovascular morbidity. This mortality rate is higher than the general population's one, but lower than the one of $40 \%$ found in another study [11] or of $75 \%$ found in a more recent one [18]. Nevertheless, the latter are probably biased by the nature of data collected. Another Italian study [17] found a prevalence of Covid-19 of $0.9 \%$ and a death rate of $0.13 \%$. Hypertension and diabetes were listed as risk factors, followed by coronary heart disease. Seventy percent of patients did not report any worsening of motor and non-motor symptoms during lockdown and this is due to paradoxical kinesia. Moreover, worsening of symptoms can be contrasted by resilience, which is enhanced in older individuals (see Table 1).

Anti-PD drugs may have a protective or detrimental role in preventing severe Covid-19. Dopamine is thought to be protective while dopa-decarboxylase is thought to be detrimental because of its co-expression with ACE2 receptor [16]. Entacapone and amantadine could act as anti-viral, [17] the latter interfering with the mechanism through which the virion enters the cell $[16,21,22]$ although in another study this was not confirmed [14].

\section{Patients' care and management}

Due to safety measures, routine follow-up visits could not take place regularly during lockdown. The role of telemedicine is, therefore, of paramount importance to prevent worsening of symptoms and preserve patients' care [17]. It can be used for follow-up and urgent visits, research, psychotherapy, genetic counselling, social services, rehabilitation and education as it has comparable outcomes to in-person visits at outpatient clinics [23]. Movement Disorders Society (MDS) has published a five-step guide for telemedicine's use in clinical practice (https://www.movementdisorders.org/ MDS/About/Committees--Other-Groups/Telemedicine-inYour-Movement-Disorders-Practice-A-Step-by-Step-Guide. $\mathrm{htm}$ ). The most important clinical scales (UPDRS, UHDRS, 
Table 1 Mortality rate and incidence rate of Covid-19 among PD patients

\begin{tabular}{lll}
\hline Study & Mortality rate & Incidence \\
\hline Antonini et al. (2020) & $40 \%, 50 \%$ in pts under advanced therapies & Only 10 pts studied \\
Fasano et al. (2020) & $5.7 \%$ (not different from general population) & $7.1 \%$ PD, 7.6\% caregivers \\
Cilia et al. (2020) & $0 \%$ & $8.4 \%$ \\
Artusi et al. (2020) & $75 \%$ PD pts, 11.53\% gen. pop & $0.57 \%$ PD patients, 0,63\% \\
& Advanced PD, nursing homes & general population \\
Del Prete et al. (2020) & $0.13 \%$ (not different from general population) & $0.9 \%$ \\
Fasano et al. (2020) & $19.2 \%$ (nature of data collection) & $/$ \\
\hline
\end{tabular}

The incidence does not differ between PD patients and general population, confirming PD itself is not a risk factor for Covid-19. Mortality rates differ between studies, in some it was similar to the general population's one, but these studies did not consider advanced PD patients and those living in nursing homes. All of this confirms PD itself is not a risk factor for severe Covid-19 and death, but advanced age and important comorbidities are
MoCA) in Movement Disorders daily practice have been validated for remote use. In a study, $51 \%$ of the patients had a device to perform visits via telemedicine, and of them $72 \%$ were able to do it [17], however in some centres, telemedicine is only limited to phone calls or email contacts [15]. A study by the MDS Epidemiology group, based on a survey sent to all MDS members, aimed to evaluate the impact of Covid-19 on access to care for patients with PD [24]. Forty-five percent of the responders said that the patients had trouble getting PD medications regularly. $69.6 \%$ of them reported increased disability, $8.4 \%$ increased hospitalization, $2.6 \%$ increased mortality and $30 \%$ felt that Covid-19 had no obvious clinical impact on PD. This survey highlights pandemics have a greater impact in poorer countries. Most of the responders that said there was no impact on accessing the medications came from countries like the US, while those who said the contrary came from Asia, Africa and South America.

Management of advanced therapies is another important issue to consider during a pandemic [15]. To ensure safety, many elective procedures have been postponed, such as new Deep Brain Stimulation (DBS) or Levodopa-Carbidopa Intestinal Gel (LCIG) implants [15]. Worsening of neurological symptoms in patients with DBS could be attributed to the bad functioning of the device, but it is advisable to check for possible infections or metabolic problems indicating the presence of Covid-19. Patients with DBS should be educated on how to use their controllers. It is mandatory to stratify the risk in case of battery substitution $[15,25]$. The substitution is urgent when the patient has elevated risk of Malignant STN DBS withdrawal syndrome, a medical emergency, threatening the patient's life, similar to the neuroleptic-induced one. It could be irreversible even with dopaminergic therapy. The patients at greater risk are those with more than 15 years of disease, more than 5 years of bilateral stimulation of subthalamic nuclei and good response to it.

There are no data to state that infusional therapies increase the risk of developing Covid-19 [15]. It is important to obtain an objective evaluation of the patient through telemedicine and to postpone the positioning of the devices. It is advisable to select the non-locked mode, to make the patient able to control the infusion. This is not possible with all patients, because some of them are at risk of developing dopamine dysregulation syndrome (DDS), more frequent in patients at risk of developing ICDs (Impulse Control Disorders) and in patients under apomorphine infusion [15]. In case of pump failure, it is important to educate the patient to always keep an emergency dosage of L-dopa and on how to get it. It is important, moreover, to educate the patient on the risk of dopamine agonist withdrawal syndrome (DAWS), which can include malignant akinesia or acute lethargy.

\section{Physiotherapy}

The positive effect of physical activity and physiotherapy on PD is well known. In 2015, Park-in-Shape study [26] demonstrated the superiority of open space activity over indoors one. Aerobic activity has also been proved superior over anaerobic one. Various studies have shown that individuals practicing moderate-intensity exercise self-report lower incidence of upper respiratory tract infections and have lower mortality rate in pneumonia, respiratory disease and aspiration pneumonia. Moderate-intensity exercise can lower neuroinflammation and improve Quality of Life (QoL) in PD patients[27]. Moreover, exercise increases neuroplasticity, via activation of brain neurotrophic factors, therefore improving cognition as well. The link between moderateintensity exercise and upper respiratory tract infections leads to hypothesize a role for exercise against Covid-19, via improving the immune response, restoring the elasticity and strength of lung tissue and respiratory muscles, reduction of ROS, and finally mitigating an individual's risk factor for sever Covid-19, such as cardiovascular comorbidities.

It was not so easy to continue practicing physiotherapy and sports during global pandemic lockdown[28]-[30]. 
Various studies have reported a dramatic reduction in physical activity due to gym closure and fear of getting Covid-19 [28]. The patients who stopped physiotherapy reported worsening of both motor and non-motor symptoms 60. In one study, although UPDRS part 3 did not differ significantly from that evaluated before lockdown, nevertheless the patients reported subjective worsening of symptoms [28].

In another study, worsening patients did not differ for age, sex, age at onset and disease duration from those referring stable conditions. but were different for the level of physical activity [30]. Some studies [31] point out that Technology Based Tools (TBTs) are feasible substitutes to gyms and outdoors activities. The most used TBTs were free video lessons, which are advisable to use and practice regularly. Virtual support groups are of paramount importance for PD patients [29], to prevent isolation and to motivate them. Advanced rehabilitation techniques are not easily accessible to all the patients [31], although there are some mobile apps than can be used as E-rehabilitation platforms. Indeed, virtual rehabilitation (VR) platforms may improve gait, balance, QoL and ADL function. Among disadvantages, patients might start to use compensatory movements to increase performance; therefore they must always be under supervision. The Engage-PD program [32] is a coaching program for newly diagnosed or early-stage PD patients. It has been rapidly adapted to the restrictive measures in the United States, therefore is has included patients with mild PD, due to the increased request of exercise during lockdown. All sessions were moved to telehealth, via Zoom meetings. Fifty-two percent of the referred patients were enrolled in the program. This was a higher rate than expected, and the authors hypothesize this is also due to parking/transportation considerations. In addition, many patients did not like to spend another hour after the visit to participate in the program, while this was not an issue for at-home exercises. The majority of patients enrolled in the program had a college or higher level of education.

Table 2 These studies explored concern of getting Covid-19, anxiety and depression in PD patients during lockdown. All of these studies reported a higher incidence of fear, anxiety and depression in patients

\begin{tabular}{llll}
\hline Study & Features analyzed & Outcome & $\begin{array}{c}\text { Perceived } \\
\text { worsening of } \\
\text { symptoms }\end{array}$ \\
\hline Stalash et al. (2020) & Depression, anxiety, QoL & $\begin{array}{c}\text { Worse in PD vs controls and in lock- } \\
\text { down vs before }\end{array}$ & / \\
Schirinzi (2020) & Anxiety, depression & Worse during lockdown \\
Salari (2020) & Anxiety & $\begin{array}{c}\text { Worse in pts vs controls and during } \\
\text { lockdown }\end{array}$ & Yes \\
Santos-Garcia et al. (2020) & Concern of getting Covid-19 & Worsening of symptoms & Yes \\
\hline
\end{tabular}

\section{Mental and cognitive health}

A case-control study [33], showed that PD patients had significantly worse stress, depression, anxiety, physical activity and quality of life than healthy controls. All these features were worse during lockdown than before. An Italian study [34] analysed the emails and phone calls to Roma Tor Vergata University: $46 \%$ of the patients searched a contact in the centre to ask about clinical services and $28 \%$ of them complained about worsening of motor or non-motor symptoms. None of them was affected by Covid-19 but they experienced worsening of the symptomatology during lockdown, consistently with the welldocumented detrimental effect of stress in PD. Fifty-two percent of them required a change in the therapy with benzodiazepines, antipsychotics or dopaminergic drugs. Seventeen percent of them, especially the younger ones, asked about the relationship between Covid-19 and PD. A questionnaire-based study [35] showed sever anxiety in $25.5 \%$ of patients and $4.8 \%$ of controls. This was severe also in $4.2 \%$ of the caregivers. People with higher education levels showed more anxiety. Anxiety did not correlate with disease duration, but related to the fear of getting Patients worried for drug availability showed higher levels of anxiety (see Table 2). The burden on caregivers is also highlighted in another study [36] and it is increased by self-isolation and closing of nursing homes. This study aimed at assessing if different PD features could differently impact on patients and caregivers' stress during lockdown and showed that the level of stress of both patients and caregivers is related to Hospital Anxiety and Depression Scale (HADS) and Non-Motor Symptoms scores (NMSS). Non-motor symptoms are known to be related to caregivers' level of stress. The home confinement period resulted in additional load of pressure on caregivers.

There are few studies on lockdown and cognition [14, 37]. One of them [37] reported a slight worsening in

than in controls, and they all pointed out that PD patients perceived a worsening of motor and non-motor symptoms, consistently with the well-known detrimental effect of stress in PD Covid-19, which was more significant in the PD group. 
mini-mental state examination, and one patient converted to dementia. The more affected clinical domains were memory and attention. Almost all patients interrupted physiotherapy and cognitive training, which are crucial to maintain cognitive health in PD.

\section{Can Covid-19 cause parkinsonism?}

Some studies have pointed out the risk of a post-Covid-19 parkinsonism [9]. So far, only three cases of parkinsonism have been reported after Covid-19 infection [38]-[40]. All three patients reported [38]-[40], were young. One of the patients experienced mild Covid-19 and bradykinesia, rigidity and tremor shortly afterwards [38]. His genetic tests for LRRK2 and GBA were negative and his F-Dopa PET scan was positive. He responded to dopaminergic therapy. The second patient was a young woman [40] who, similarly to the patient reported previously [38], experienced mild Covid-19 and shortly afterward presented with acute rigid-akinetic parkinsonism. She also had reduced dopamine reuptake in the basal ganglia (left putamen) and as the other patient [38] responded to dopaminergic therapy, but she was not tested for any genetic mutation. The third case [39] is different from the previous ones. He experienced severe Covid-19 and was treated in Intensive Care Unit shortly after admission. He needed mechanical ventilation for 23 days and started to present with myoclonic jerks. After two episodes of decreased consciousness, he presented with an opsoclonus/myoclonus syndrome. After he recovered, he presented with hyposmia, vertical gaze impairment with round-the house sign, impaired smooth pursuit and intermittent opsoclonus and a right-dominant rigid-hypokinetic syndrome. His DAT-scan was positive for reduced dopamine uptake on both sides, worse in the left putamen. He had no family history. He did not respond to dopaminergic therapy, but some spontaneous improvement and recovery was observed after some time, to the point the patient was able to take a few steps on his own.

There are some possible mechanisms involved [9]:

- Acute structural or functional damage of the basal ganglia;

- Extensive hypoxic or inflammatory brain injury due to an encephalopathy;

- Unmasking of un underlying, but previously unknown, parkinsonism;

- A viral long-term damage to basal ganglia which after some time might induce parkinsonism.

The first two and the last possibilities imply that there is a structural or functional damage of the basal ganglia, and this causes a clinical and anatomopathological disease which is different from Parkinson's disease. After 1918 Spanish flu pandemic, the world witnessed the spreading of a post-encephalitic parkinsonism [13]. The patients with post-encephalitic parkinsonism described by Von Economo experienced oculogyric crisis, pupillary disturbances, alteration of sleep and psychiatric symptoms [9], and the parkinsonism developed years later. Moreover, in the brains of such patients there were not Lewy bodies, which are the hallmark of Parkinson's Disease, and neurofibrillary tangles were observed in the absence of neuritic plaques [9]. Influenza viruses are neurotropic, and Sars-Cov2 neurotropism has not yet been proved, hence suggesting a possible immune-mediated mechanism [9]. The mechanism could be a disrupted blood-brain barrier due to a cytokine storm, bur nevertheless it is not demonstrated that Sars-Cov2 has a special tropism for substantia nigra or basal ganglia [9]. Therefore, on such basis, we might conclude that only one patient [39] of the three reported [38]-[40] had a Covid19-associated encephalopathy, which, through an immunemediated mechanism may have induced a rigid-akinetic syndrome, which was not responsive to levodopa and like to Von Economo's cases. The other two patients [38, 40] did not have an encephalopathy, and developed a dopaminergicresponsive parkinsonism.. Here the more likely responsible mechanism in these two cases is an unmasking of an already latent Parkinson's Disease.

It is too early to be conclusive on the matter "Can Covid19 cause parkinsonism" but we might be able to answer the question in the next few years.

\section{Conclusion}

Covid-19 has a great impact on PD patients, exacerbating motor and non-motor symptoms. PD patients are not at greater risk of developing severe Covid-19, though cardiovascular comorbidities do have a role. The profound impact of Covid 19 must be acknowledged to improve the overall patient's clinical care. Due to lockdown restrictions, telehealth should be promoted whenever possible encouraging routine telemedicine visits and e-rehabilitation sessions. Special attention should be reserved to caregivers' burden promoting their education and training as well as their psychophysical health. Further research is needed to better understand the possible link between Sars-Cov2 and post-encephalitic parkinsonism.

Authors' contribution Not relevant.

Funding No funding was received to assist in the preparation of this manuscript.

Availability of data and material Not relevant. 


\section{Declarations}

Conflicts of interest The Authors have no disclosure to declare.

\section{References}

1. Wiersinga WJ, Rhodes A, Cheng AC, Peacock SJ, Prescott HC (2020) Pathophysiology, transmission, diagnosis, and treatment of coronavirus disease 2019 (COVID-19): a review. JAMA 324(8):782-793. https://doi.org/10.1001/jama.2020.12839

2. Meng $L$ et al (2020) Intubation and ventilation amid the COVID19 outbreak: Wuhan's experience. Anesthesiology. https://doi.org/ 10.1097/ALN.0000000000003296

3. Chen N, Zhou M, Dong X, Qu J, Gong F, Han Y, Qiu Y, Wang J, Liu Y, Wei Y, Yu T (2020) Epidemiological and clinical characteristics of 99 cases of 2019 novel coronavirus pneumonia in Wuhan, China: a descriptive study. The Lancet 395(10223):507-13

4. Harenberg J, Favaloro E (2020) COVID-19: Progression of disease and intravascular coagulation-present status and future perspectives. Clin Chem Lab Med 58(7):1029-1036. https://doi.org/ 10.1515/cclm-2020-0502

5. Iba T, Levy JH, Levi M, Thachil J (2020) Coagulopathy in COVID-19. J Thromb Haemost 18(9):2103-2109. https://doi. org/10.1111/jth.14975

6. Jose RJ, Manuel A (2020) COVID-19 cytokine storm: the interplay between inflammation and coagulation. Lancet Respir Med 8(6):e46-e47. https://doi.org/10.1016/S2213-2600(20)30216-2

7. Asadi-Pooya AA, Simani L (2020) Central nervous system manifestations of COVID-19: a systematic review. J Neurol Sci. https:// doi.org/10.1016/j.jns.2020.116832

8. Andalib S et al (2021) Peripheral nervous system manifestations associated with COVID-19. Curr Neurol Neurosci Rep 21(3):2021. https://doi.org/10.1007/s11910-021-01102-5

9. Merello M, Bhatia KP, Obeso JA (2021) SARS-CoV-2 and the risk of Parkinson's disease: facts and fantasy. Lancet Neurol 20(2):94-95. https://doi.org/10.1016/S1474-4422(20)30442-7

10. Bhidayasiri R, Virameteekul S, Kim J-M, Pal PK, Chung S-J (2020) COVID-19: an early review of its global impact and considerations for Parkinson's Disease patient care. J Movement Disord. https://doi.org/10.14802/jmd.20042/J

11. Antonini A, Leta V, Teo J, Chaudhuri KR (2020) Outcome of Parkinson's disease patients affected by COVID-19. Movement Disord 35(6):905-908. https://doi.org/10.1002/mds.28104

12. Ait Wahmane S, Achbani A, Ouhaz Z, Elatiqi M, Belmouden A, Nejmeddine $M$ (2020) The possible protective role of $\alpha$-Synuclein against severe acute respiratory syndrome Coronavirus 2 infections in patients with Parkinson's Disease. Movement Disord 35(8):1293-1294. https://doi.org/10.1002/mds.28185

13. Sulzer D et al (2020) COVID-19 and possible links with Parkinson's disease and parkinsonism: from bench to bedside. NPJ Parkinson's Disease. https://doi.org/10.1038/s41531-020-00123-0

14. Cilia R et al (2020) Effects of COVID-19 on Parkinson's Disease clinical features: a community-based case-control study. Mov Disord 35(8):1287-1292. https://doi.org/10.1002/mds.28170

15. Fasano A et al (2020) Management of advanced therapies in parkinson's disease patients in times of humanitarian crisis: The COVID-19 experience. Movement Disord Clin Pract 7(4):361372. https://doi.org/10.1002/mdc3.12965

16. Fasano A et al (2020) Predictors of COVID-19 outcome in Parkinson's disease. Park Relat Disord 78:134-137. https://doi.org/ 10.1016/j.parkreldis.2020.08.012

17. Del Prete E et al (2020) Prevalence and impact of COVID19 in Parkinson's disease: evidence from a multi-center survey in Tuscany region. J Neurol. https://doi.org/10.1007/ s00415-020-10002-6

18. Artusi CA et al (2020) COVID-19 in Parkinson's disease: report on prevalence and outcome. Parkinsonism Relat Disord 80:7-9. https://doi.org/10.1016/j.parkreldis.2020.09.008

19. Artusi CA et al (2021) COVID-19 and Parkinson's disease: what we know so far? J Dis Parkinsons. https://doi.org/10.3233/ jpd-202463

20. Fasano A et al (2020) COVID-19 in Parkinson's Disease Patients Living in Lombardy, Italy. Mov Disord 35(7):1089-1093. https:// doi.org/10.1002/mds.28176

21. Smieszek S, Przychodzen B, Polymeropoulos M (2020) Amantadine disrupts lysosomal gene expression; potential therapy for COVID19. BioRxiv. https://doi.org/10.1101/2020.04.05.026187

22. Kobylecki C, Jones T, Lim CK, Miller C, Thomson AM (2020) Phenomenology and outcomes of in-patients with parkinson's disease during the Coronavirus Disease 2019 pandemic. Movement Disord 35:1295-1296. https://doi.org/10.1002/mds.28205

23. Cubo E, Hassan A, Bloem BR, Mari Z (2020) Implementation of telemedicine for urgent and ongoing healthcare for patients with Parkinson's disease during the COVID-19 pandemic: new expectations for the future. J Parkinson's Dis 10(3):911-913. https:// doi.org/10.3233/JPD-202108

24. Cheong JLY, Goh ZHK, Marras C, Tanner CM, Kasten M, Noyce AJ (2020) The impact of COVID-19 on access to Parkinson's disease medication. Mov Disord 35(12):2129-2133. https://doi. org/10.1002/mds.28293

25. Miocinovic $\mathrm{S}$ et al (2020) Recommendations for Deep brain stimulation device management during a pandemic. J Parkinson's Dis. https://doi.org/10.3233/JPD-202072

26. van der Kolk NM et al (2015) Design of the Park-in-Shape study: A phase II double blind randomized controlled trial evaluating the effects of exercise on motor and non-motor symptoms in Parkinson's disease. BMC Neurol. https://doi.org/10.1186/ s12883-015-0312-6

27. Van De Wetering-Van Dongen VA, Kalf JG, Van Der Wees PJ, Bloem BR, Nijkrake MJ (2020) The effects of respiratory training in Parkinson's disease: a systematic review. J Parkinson's Dis. https://doi.org/10.3233/JPD-202223

28. Song J, Ahn JH, Choi I, Mun JK, Cho JW, Youn J (2020) The changes of exercise pattern and clinical symptoms in patients with Parkinson's disease in the era of COVID-19 pandemic. Park Relat Disord 80:148-151. https://doi.org/10.1016/j.parkreldis.2020.09. 034

29. Subramanian I (2020) Virtual Parkinson's disease support groups in the COVID-19 Era: social connection in the time of social distancing. Movement Disord Clin Pract 7(6):739-740. https://doi. org/10.1002/mdc3.12994

30. Schirinzi T et al (2020) Physical activity changes and correlate effects in patients with Parkinson's disease during COVID-19 lockdown. Mov Disord Clin Pract 7(7):797-802. https://doi.org/ $10.1002 / \mathrm{mdc} 3.13026$

31. Srivastav AK, Samuel AJ (2020) E-rehabilitation: one solution for patients with Parkinson's disease in COVID-19 era. Parkinsonism Relat Disord 75:128-129. https://doi.org/10.1016/j.parkr eldis.2020.05.021

32. Shah H, Quinn L, Macpherson C, Long K, Shah H (2020) Promoting physical activity via telehealth in people with Parkinson Disease: the path forward after the COVID-19 pandemic?". Phys Ther 100:1-7. https://doi.org/10.1093/ptj/pzaa128/5876269

33. Shalash A et al (2020) mental health, physical activity, and quality of life in parkinson's disease during COVID-19 pandemic. Movement Disord 35(7):1097-1099. https://doi.org/10.1002/mds.28134

34. Schirinzi T et al (2020) Self-reported needs of patients with Parkinson's disease during COVID-19 emergency in Italy. Neurol Sci 41(6):1373-1375. https://doi.org/10.1007/s10072-020-04442-1 
35. Salari M et al (2020) Incidence of anxiety in parkinson's disease during the coronavirus disease (COVID-19) pandemic. Movement Disord 35(7):1095-1096. https://doi.org/10.1002/mds.28116

36. Oppo V et al (2020) Parkinson's Disease symptoms have a distinct impact on caregivers' and patients' stress: a study assessing the consequences of the COVID-19 lockdown. Movement Disord Clin Pract 7(7):865-867. https://doi.org/10.1002/mdc3.13030

37. Palermo G, Tommasini L, Baldacci F, Del Prete E, Siciliano G, Ceravolo R (2020) Impact of Coronavirus Disease 2019 pandemic on cognition in Parkinson's Disease. Movement Disord 35(10):1717-1718. https://doi.org/10.1002/mds.28254
38. Mikhal E. Cohen GY, Roni E, Bettina S-B, Amir J, Maxim I, Rachel BS, Jefri JP, Hanaa G, Volha S, Natan M (2020) A case of Parkinson disease after SARS-CoV-2 infection. Lancet Neurol

39. Méndez-Guerrero A et al (2020) Acute hypokinetic-rigid syndrome following SARS-CoV-2 infection. Neurology 95(15):e2109-e2118. https://doi.org/10.1212/WNL.0000000000 010282

40. Faber I, Brandão PRP, Menegatti F, de Carvalho Bispo DD, Maluf FB, Cardoso F (2020) Coronavirus disease 2019 and Parkinsonism: a non-post-encephalitic case. Mov Disord 35(10):1721-1722. https://doi.org/10.1002/mds.28277 